\title{
Communicating prostate cancer risk: what should we be telling our patients?
}

\author{
Peter D Baade, Suzanne K Steginga, Carole B Pinnock and Joanne F Aitken
}

W Thile we wait for definitive evidence of the effectiveness of screening and treatment strategies for prostate cancer, men are asking for guidance on whether they should be screened for this cancer. Personal vulnerability and perceived seriousness of the disease influence this decision, ${ }^{1}$ and population-based risks are often quoted in decision-support material for this purpose. ${ }^{2}$ We suggest that the oft-used statement "men are more likely to die with prostate cancer than from prostate cancer" may be incorrect for men diagnosed in their 50s and 60s, and that doctors need to individualise the risk of prostate cancer for their patients.

We use Australian data and published reports to show that the use of routine population risk statistics can be misleading, and suggest methods to make this risk information more patient specific. We do not address risks associated with treatment choices, nor the best methods of presenting risks. ${ }^{3,4}$

\section{Relevance of personal risk}

There is considerable debate about whether screening for prostate cancer is beneficial and whether opportunistic screening should be offered. $^{5,6}$ The current clinical advice in Australia is not to recommend population-based screening of asymptomatic men for prostate cancer. ${ }^{7-9}$ However, most guidelines advocate that men should be able to access screening so long as they are fully informed of the potential risks, benefits and uncertainties of investigations and treatment. A man's perception of his personal risk and vulnerability to prostate cancer has been shown to influence his decision about whether to be tested. ${ }^{1}$ In addition, because of short- and long-term implications for quality of life, men need to be supported to apply their own personal values or preferences in relation to those risks when they decide about testing (see Box 1).

\section{What do we know about population risk estimates?}

Taking standard population risk estimates (Box 2) at face value, it seems reasonable to suggest to patients that being diagnosed with prostate cancer is very rare among men younger than 50 and that mortality is very rare among men younger than 60 years. Men are much more likely to die from heart disease, and to a lesser extent lung cancer, than from prostate cancer. However, there are a

\footnotetext{
Queensland Cancer Fund, Spring Hill, QLD.

Peter D Baade, PhD, Senior Research Fellow, Viertel Centre for

Research in Cancer Control; Suzanne K Steginga, PhD, Director,

Community Services; Joanne F Aitken, PhD, Director, Viertel Centre for Research in Cancer Control.

Repatriation General Hospital, Daw Park, SA.

Carole B Pinnock, PhD, Principal Research Scientist, Urology Unit.

Reprints: Dr P D Baade, Queensland Cancer Fund, GPO Box 201, Spring

Hill, QLD 4004. pbaade@qldcancer.com.au
}

\section{ABSTRACT}

- Until definitive evidence of the effectiveness of prostate cancer screening is available, most guidelines advocate that men make their own decisions about testing, after being fully informed. A man's perception of his personal risk is a key element in the decision-making process. In this decisionmaking, the current routine use of population risk estimates may be misleading.

- Risk estimates need to be relevant to the man making the choice. In particular, they should be age-specific and, where possible, include adjustments for known risk factors such as family history. As an example, although the population risk of lung cancer mortality is twice that of prostate cancer, for a non-smoking man with a family history of prostate cancer the direction of this comparison would be reversed.

- A man aged 50 diagnosed with prostate cancer has a greater likelihood (60\%) of dying prematurely (before 80 years) from prostate cancer than a man diagnosed when aged 70 (38\%). This can be attributed to the longer time available for the prostate cancer to progress, and the increased effect of competing causes of death among older men.

- This suggests that the oft-used statement "men are more likely to die with prostate cancer than from prostate cancer" is misleading, particularly for men diagnosed in their 50 s or 60s.

- Decisions need to be made by men based on the best possible understanding of their personal vulnerability, and the individualisation of risk provides a more realistic appraisal of potential threat posed by the disease.

MJA 2005; 182: 472-475

number of reasons why routinely available population risk information may give an incomplete perspective.

\section{Risk estimates need to be age-appropriate}

The relative population risk of conditions varies depending on the age range examined. When presenting risk estimates, relevant age ranges need to be used. For example, a 50-year old man presented with a risk table for men aged 0-75 would note that that colorectal cancer mortality was twice as likely as death due to prostate cancer. However, for a man aged 50 who is interested in assessing his future risk of dying from prostate cancer, the risk for men younger than 50 is not relevant. Including it could result in a spuriously low estimate of population risk for prostate cancer relative to other conditions. Similarly, a man currently aged 50 has at least 30 years of additional life expectancy. ${ }^{15}$ Truncating risk estimates at 75 years would also underestimate his true risk of premature mortality. 


\section{FOR DEBATE}

\section{Decision steps for informed choice about prostate- specific antigen testing in asymptomatic men ${ }^{10}$}

1. Identify the patient's main concern

2. Explain where the prostate is and the tests available to detect prostate cancer

3. Discuss prostate cancer risk and risk factors

4. Explain the pros and cons of early detection of prostate cancer

5. Identify patient's personal preferences

6. Support the patient's choice, and if requested implement a prostate cancer risk management plan

\section{Personal risk depends on other factors}

Apart from age, other factors increase prostate cancer incidence risk. Having a first-degree relative (ie, brother or father) with prostate cancer diagnosed at an early age (under 60 years) at least doubles a man's risk of being diagnosed with prostate cancer. ${ }^{16}$ The risk increase is highest in relatives of men diagnosed before age 60 , and decreases with increasing age of the affected relative. ${ }^{16}$ These estimates are primarily based on invasive prostate cancers, ${ }^{16}$ and so cannot be explained by increased likelihood of relatives having prostate-specific antigen (PSA) tests. Although there is a risk increase for African Americans ${ }^{17}$ there is no evidence of a similar increase in risk for Indigenous Australian men. ${ }^{18}$

We also know that some men are more likely to get certain conditions because they are exposed to known risk factors for that disease. For example, latest estimates suggest that a current smoker is 6 to 15 times more likely to develop lung cancer than a non-smoker. ${ }^{19}$ Combining this with at least a twofold increase in prostate cancer diagnosis risk for a man with a family history of prostate cancer, ${ }^{16}$ we could reasonably suggest that a nonsmoking man with a family history of prostate cancer would be at a greater risk of dying from prostate cancer than of lung cancer. This is a much different scenario to that implied earlier (Box 2).

There are also unknown factors. The wide international variability in incidence rates of prostate cancer, ${ }^{17}$ and increased rates among second-generation Japanese migrants to the United States compared with Japanese men living in Japan ${ }^{20}$ suggest there may also be environmental or dietary exposures that influence prostate cancer risk. Unfortunately, it is currently unclear what specific factors are involved, and the level to which they affect an individual's risk. ${ }^{21}$

\section{Description of analytical strategy}

Mortality data for Australian men were obtained from the Australian Bureau of Statistics. Data on the number of new prostate cancers diagnosed among Australian men were obtained from the Australian Institute of Health and Welfare for the period 1997 to 2001 (unpublished cancer incidence data, February 2005). ${ }^{11}$

Age-specific risks of being diagnosed with, or dying from, prostate cancer were calculated using cumulative rates (risk per 1000 men). These risks represent the risk of a man at the start of the age group moving through to the end of that age group, experiencing the current age-specific rates. To maintain comparability between incidence and mortality, risks of dying from prostate cancer were also calculated using 1997 to 2001 data (unpublished cancer mortality data, February 2005)..$^{12}$ However, as Australian mortality data were available to $2002{ }^{12}$ mortality risk differentials across a range of conditions were calculated using 1999-2002 mortality data.

The ICD-10 codes for the main causes of death considered in this analysis were heart disease (I05-109, 111, I13, 121-I26), lung cancer (C34), colorectal cancer (C18-C20), prostate cancer (C61), stroke (160-169, G45, G46), chronic obstructive pulmonary disease (I27, J67, J40-J44) and accidents (V01-X59). Additional Queensland data looking at cause-specific survival were obtained for prostate cancers diagnosed between 1982 and 2000 , with follow-up to 2002 (Queensland Health, unpublished data, 2005). Because of a perceived difficulty in establishing the cause of death among the oldest of the elderly, ${ }^{13}$ we limited this analysis to men aged 50-79 years. The 10-year cause-specific survival estimates were calculated using PROC LIFETEST in SAS. ${ }^{14}$ Extrapolations of the cause-specific survival curves (to a maximum of 30 years after diagnosis) were made using exponential regression models. Population risk estimates

Current population risk estimates of Australian men being diagnosed with prostate cancer or dying from prostate cancer are shown below. For all ages, the risk of dying from prostate cancer is less than the risk of being diagnosed with prostate cancer, while both the diagnosis risk and mortality risk increase with age.

Population risks of prostate cancer diagnosis and death, Australia 1997-2001 (per 1000 men)

\begin{tabular}{lcc} 
Age group (years) & Risk of diagnosis & Risk of death \\
\hline$<40$ & $<0.1$ & $<0.01$ \\
$40-49$ & 1 & $<0.1$ \\
$50-59$ & 12 & 1 \\
$60-69$ & 45 & 5 \\
$70-79$ & 79 & 21 \\
$80-89$ & 105 & 61 \\
$0-79$ & 137 & 27 \\
$50-79$ & 136 & 27
\end{tabular}

Population risk of death for 50 -year-old Australian men to age 79 by leading causes of death (1999-2002)

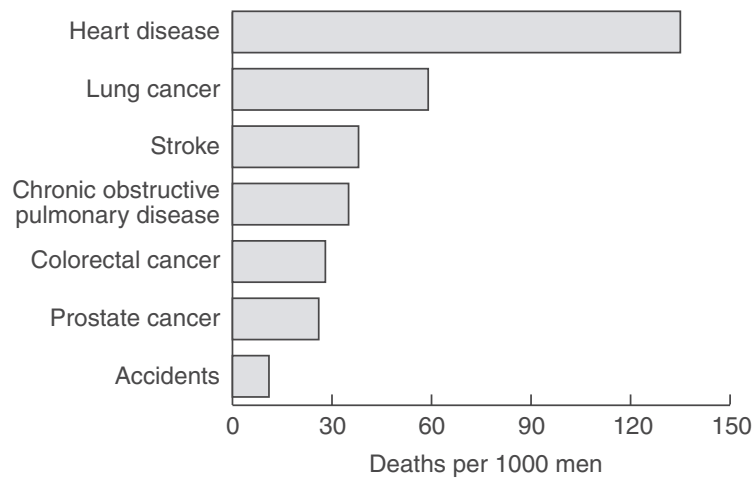




\section{Mortality risk changes after diagnosis}

Population mortality risk estimates do not distinguish between men who already have a diagnosis of prostate cancer and those who do not. The risk of dying from prostate cancer depends on the stage at which the cancer was diagnosed, ${ }^{22}$ and the Gleason score. ${ }^{23}$ Unfortunately, these prognostic indicators are not routinely collected in Australia and it is not clear how international data can be generalised to Australia. Combining all prostate cancers (regardless of stage and Gleason score), 5-year relative survival from prostate cancer has been shown to reduce slightly as age of diagnosis increases. ${ }^{24}$ However, 5 -year survival data may not provide a complete picture of risk, as it has been suggested that initially indolent cancers can progress into metastatic disease in the long term. ${ }^{25}$

\section{Men diagnosed with prostate cancer at a young age are more likely to die prematurely}

The implications of a diagnosis of prostate cancer among men in their 50s need to include the greater average life expectancy of men in their 50s compared with men in their 70s. One way of doing this is to consider the expected proportions of men still alive at 80 years of age (which is close to the current life expectancy of men at birth ${ }^{15}$ ). For Queensland men diagnosed in their 50s, the projected 30-year cause-specific survival was 0.399. The 20-year cause-specific survival for men diagnosed in their 60s was 0.497 , and the 10-year cause-specific survival for men diagnosed in their 70 s was 0.618 . Therefore, about three-fifths (60\%) of men diagnosed at 50 could be expected to die a premature death (ie, before reaching 80 years of age) from prostate cancer. Corresponding percentages for premature death for men diagnosed at 60 and 70 years are $50 \%$ and $38 \%$.

\section{Competing causes of death for men diagnosed with prostate cancer}

Based on Queensland data (Queensland Health, unpublished data, 2005), for each 100 men diagnosed in their 50s, about 30 had died from their prostate cancer within 10 years, and an additional eight died from other causes within the same period (Box 3). The corresponding values for each 100 men diagnosed in their 70s were 40 and 29. Therefore, even though the numbers dying from prostate cancer within 10 years were similar, men diagnosed at a later age were nearly four times more likely to have died from other causes within 10 years.

Combined, these figures highlight the observation that for a 50year-old man, a diagnosis of prostate cancer is more likely to result in premature death from the disease than for men diagnosed in their 70s. This is due both to the longer average life expectancy of younger men and to the higher impact of other causes of death as men get older. These results suggest that the often-used statement "men are more likely to die with prostate cancer than from prostate cancer" is misleading, particularly for men diagnosed in their $50 \mathrm{~s}$ and 60s.

\section{Is knowledge of increased risk of premature death useful?}

Should the relatively increased risk of dying prematurely after a prostate cancer diagnosis for men in their 50 s be communicated to men who are making this health decision? Views here will differ depending on the life experience and background of the individual. Some may take the view that until we have definitive evidence that PSA testing and active treatment has an effect on patient outcomes, knowledge about age-specific mortality is unimportant. However, most studies indicate that personal risk from, or vulnerability to, disease is perceived as an important factor by men making a screening decision.,26,27 For men who are actually diagnosed with localised prostate cancer, the desire for cure and fear of cancer spread strongly influences subsequent treatment choices, and most men with localised disease choose active treatment, ${ }^{28}$ despite the lack of definitive proof that treatment extends life. Men who choose early detection are asking for the opportunity to have that choice. Access to the best possible information on personal vulnerability to disease is consistent with medicolegal requirements for providing patient information. ${ }^{29}$ We suggest this includes population-based estimates of risk of premature death given his age at diagnosis, and other relevant risk factors.

\section{PSA testing may be affecting the observed risk estimates}

Population incidence and premature-mortality risk estimates relate to diagnosed prostate cancer only. Based on autopsy results,

\section{Comparison of 10-year cause-specific survival estimates by age at diagnosis}

One hundred men diagnosed in their 50s: 10 years later

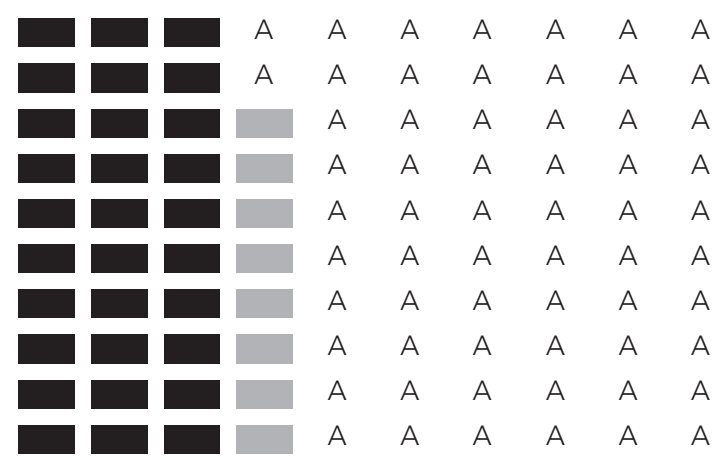

One hundred men diagnosed in their 70s: 10 years later

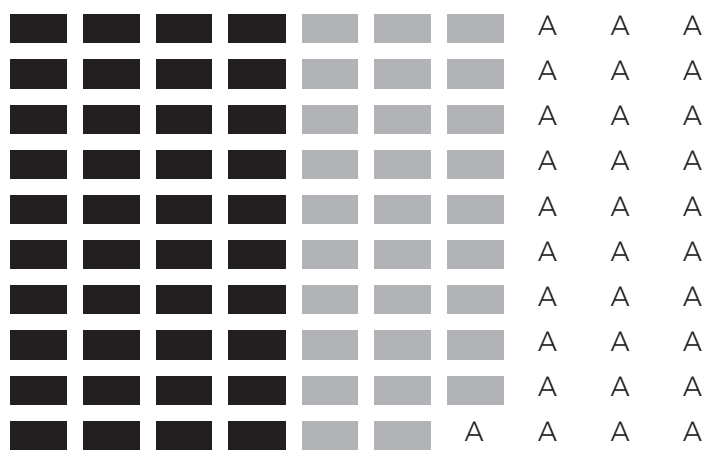


significant proportions of men may have undiagnosed histological prostate cancer, even though it may not have caused symptoms or death. ${ }^{30}$ It is likely that, with the advent of PSA testing, some of these latent prostate cancers are now being diagnosed. It is likely that current calculations overestimate the real incidence risk and underestimate the real premature mortality risk by including these PSA-detected latent prostate cancers. As we cannot yet distinguish between those patients who have latent cancers and those men whose prostate cancer has potential to cause mortality, ${ }^{31}$ we need to accept this limitation for current estimates.

\section{Should we wait for definitive data?}

The only study design that can definitively measure whether prostate cancer screening reduces mortality on a population basis, and at what cost in terms of financial costs and quality of life, is a randomised controlled trial. These trials are currently under way in Europe and the United States, ${ }^{32,33}$ but the first results may not be available for several years. However, the absence of certainty does not necessarily imply the absence of an effect. Screening may ultimately be shown to prevent men dying from prostate cancer, and, paradoxically, while prostate cancer is less common in younger men, it is these men who may benefit most.

It is also clear that, even if evidence of effectiveness is available, men will still face a dilemma in trading off the possible immediate quality-of-life costs of treatment if prostate cancer is detected against an uncertain longer term survival gain. Thus, a process of understanding and evaluating personal risk will be an essential component of informed choice for prostate cancer testing for some time to come.

\section{Competing interests}

None identified.

\section{References}

1 Wolf AM, Philbrick JT, Schorling JB. Predictors of interest in prostatespecific antigen screening and the impact of informed consent: what should we tell our patients? Am J Med 1997; 103: 308-314.

2 Gattellari M, Ward J. Does evidence-based information about screening for prostate cancer enhance consumer decision making? A randomised controlled trial. J Med Screen 2003; 10: 27-39.

3 Gigerenzer G, Edwards A. Simple tools for understanding risks: from innumeracy to insight. BMJ 2003; 327: 741-744.

4 Paling J. Strategies to help patients understand risks. BMJ 2003; 327: 745-748.

5 Frankel S, Smith GD, Donovan J, et al. Screening for prostate cancer. Lancet 2003; 361: 1122-1128.

6 Bunting PS. Screening for prostate cancer with prostate-specific antigen: beware the biases. Clin Chim Acta 2002; 315: 71-97.

7 Prostate cancer screening: summary of the review prepared by the Australian Health Technology Advisory Committee. Canberra: Commonwealth Department of Health and Family Services, 2003. Available at: http://www7.health.gov.au/pubs/ahtac/prostate.htm (accessed Mar 2005).

8 Guidelines for preventive activities in general practice (Updated 5th edition). Aust Fam Physician 2002; (Special issue - May 2002).

9 The Cancer Council Australia. National cancer prevention policy 200406. Sydney: Cancer Council Australia, 2004. Available at: http://www.can-
cer.org.au/documents/NatCancerPreventPol_04-06.pdf (accessed Dec 2004).

10 Steginga SK, Pinnock C, Baade PD. The early detection of prostate cancer in general practice: supporting patient choice. Practice resource in supporting patients' choice about PSA testing in general practice. Brisbane: Queensland Cancer Fund, 2005.

11 Cancer in Australia 2001. Canberra: Australian Institute of Health and Welfare and Australasian Association of Cancer Registries, 2004. (AlHW Catalogue No. CAN 23.)

12 Australian Bureau of Statistics. Causes of death (various years). Canberra: ABS, 2004. (ABS Catalogue No. 3303.0.)

13 Grulich AE, Swerdlow A, dos Santos Silva I, et al. Is the apparent rise in cancer mortality in the elderly real? Analysis of changes in certification and coding of cause of death in England and Wales, 1970-1990. Int J Cancer 1995; 63: 164-168.

14 SAS OnlineDoc [computer program]. Version 9.1.2. Cary, NC: SAS Institute Inc, 2004.

15 Australian Bureau of Statistics. Life tables Australia 2001-2003. Canberra: ABS, 2004. (ABS Catalogue No. 3302.0.55.001.)

16 Johns LE, Houlston RS. A systematic review and meta-analysis of familial prostate cancer risk. BJU Int 2003; 91: 789-794.

17 Parkin DM, Whelan SL, Ferlay J, et al, editors. Cancer incidence in five continents. Volume VIII. IARC Scientific Publications No 155. Lyon, France': International Agency for Research on Cancer (WHO) and International Association of Cancer Registries, 2002.

18 Condon JR, Armstrong BK, Barnes A, et al. Cancer in Indigenous Australians: a review. Cancer Causes Control 2003; 14: 109-121.

19 Clinical practice guidelines for the prevention, diagnosis and management of lung cancer. Sydney: The Cancer Council Australia, 2004.

20 Leach R, Pollock B, Basler J, et al. Chemoprevention of prostate cancer: focus on key opportunities and clinical trials. Urol Clin North Am 2003; 30: 227-237.

21 Gronberg H. Prostate cancer epidemiology. Lancet 2003; 361: 859-864.

22 Jemal A, Clegg LX, Ward E, et al. Annual report to the nation on the status of cancer, 1975-2001, with a special feature regarding survival. Cancer 2004; 101: 3-27.

23 DeMarzo AM, Nelson WG, Issacs WB, et al. Pathological and molecular aspects of prostate cancer. Lancet 2003; 361: 955-964.

24 Cancer survival in Australia 2001: Part 2 Statistical tables. Canberra: Australian Institute of Health and Welfare and Australasian Association of Cancer Registries, 2001. (AlHW Catalogue No. CAN 14.)

25 Johansson JE, Andren O, Andersson SO, et al. Natural history of early, localized prostate cancer. JAMA 2004; 291: 2713-2719.

26 Weller D, Pinnock C, Silagy C, et al. Prostate cancer testing in SA men: influence of sociodemographic factors, health beliefs and LUTS. Aust NZ J Public Health 1998; 22(3 Suppl): 400-402.

27 Ward JE, Hughes AM, Hirst GHL, et al. Men's estimate of prostate cancer risk and self-reported rates of screening. Med J Aust 1997; 167: 250-253.

28 Steginga SK, Occhipinti S, Gardiner RA, et al. Making decisions about treatment for localised prostate cancer. BJU Int 2002; 89: 255-260.

29 Pinnock CB. PSA testing in general practice: can we do more now [conference report]. Med J Aust 2004; 180: 379-381.

30 Sakr WA, Haas GP, Cassin BF, et al. The frequency of carcinoma and intraepithelial neoplasia of the prostate in young male patients. $J$ Urol 1993; 150: 379-385.

$31 \mathrm{Klotz}$ LH. Active surveillance with selective delayed intervention: a biologically nuanced approach to favorable-risk prostate cancer. Clin Prostate Cancer 2003; 2: 106-110.

32 Standaert B, Denis L. The European randomised study of screening for prostate cancer. Cancer 1997; 80: 1830-1834.

33 De Koning HJ, Auvinen A, Sanchez AB, et al. Large-scale randomized prostate cancer screening trials: program performances in the European randomized screening for prostate cancer trial and the prostate, lung, colorectal and ovary cancer trial. Int J Cancer 2002; 97: 237-244.

(Received 20 Aug 2004, accepted 4 Mar 2005) 